\title{
Actuaciones docentes consideradas como incorrectas por los alumnos de Medicina: análisis comparativo entre dos universidades ${ }^{1}$
}

\author{
Ana-María Rancich, Luciana Yesica Niz, María Paula Caprara, María Eugenia Aruanno, \\ Martín Donato, Miguel-Ángel Sánchez González y Ricardo Jorge Gelpi
}

\begin{abstract}
Resumen
El objetivo del trabajo fue analizar y comparar qué actos incorrectos realizados por los docentes y sus motivos percibieron los alumnos de primer año de Medicina de las universidades de Buenos Aires (UBA) y Complutense de Madrid (UCM), utilizando una encuesta con ítems cerrados y abiertos, con el fin de narrar el incidente e indicar su motivo. Las categorías del incidente más señaladas en ambos grupos fueron: maltrato verbal, desinterés por el alumno y evaluación injusta. Los motivos más indicados: soberbia y falta de compromiso. E1 79.1\% de los alumnos de la UCM manifestaron que el incidente ocurrió en enseñanza-aprendizaje; y el 68.1\% de los de la UBA, en evaluación. Es interesante observar que en ambas universidades los estudiantes percibieron maltrato, a pesar de que no se preguntó sobre ello. Los resultados indicarían la necesidad de reforzar el compromiso de los docentes en cuanto a respeto, responsabilidad y justicia.
\end{abstract}

Palabras clave: relación docente-alumno, educación médica, principalismo.

Ana-María Rancich

arancich@fmed.uba.ar Doctora en Humanidades Médicas, Facultad de Medicina, Universidad de Buenos Aires (UBA), Argentina; profesional de apoyo a la Investigación del Consejo Nacional de Investigaciones Científicas y Técnicas (CONICET), Argentina. Temas de investigación: relación ética docente-alumno en educación médica y análisis de juramentos médicos.

Luciana-Yesica Niz

luciananiz@hotmail.com

Médica, Facultad de Medicina, Universidad de Buenos Aires, Argentina; pasante del Instituto de Fisiología Cardiovascular, UBA.

\section{María-Paula Caprara}

Médica, Facultad de Medicina, Universidad de Buenos Aires, Argentina; pasante del Instituto de Fisiología Cardiovascular, UBA.

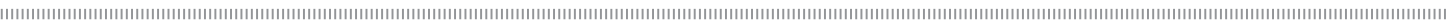

${ }^{1}$ Los autores agradecen a la doctora Valeria A. Forlizzi, profesora adjunta a cargo, y al doctor Bruno Buchholz, jefe de Trabajos Prácticos, ambos del Departamento de Anatomía, Primera Cátedra de la Universidad de Buenos Aires, por permitir administrar la encuesta a los alumnos. Asimismo, agradecen a doctora Paloma Astasio Arbiza, directora del Departamento de Medicina Preventiva y Salud Pública (Historia de la Ciencia), Facultad de Medicina, Universidad Complutense de Madrid, España, por permitir administrar la encuesta a los alumnos.
} 


\section{Martín Donato}

mdonato@fmed.uba.ar

Doctor en Medicina, Facultad de Medicina, Universidad de Buenos Aires, Argentina; investigador adjunto CONICET, Argentina. Temas de investigación: relación ética docente-alumno en educación médica y fisiopatología cardiovascular.

Miguel-Ángel Sánchez-González

migsan@med.ucm.es Doctor en Medicina, Facultad de Medicina, Universidad Complutense de Madrid (UCM), España; profesor titular de Historia de la Ciencia, UCM. Temas de investigación: bioética y métodos de investigación en bioética.

Ricardo Jorge Gelpi rgelpi@fmed.uba.ar Doctor en Medicina, Facultad de Ciencias Médicas, Universidad Nacional de La Plata, Argentina; investigador independiente, CONICET. Temas de investigación: relación ética docente-alimno en educación médica y fisiopatología cardiovascular.

\section{Atuações docentes consideradas como incorretas pelos alunos de medicina: análise comparativa entre duas universidades}

\section{Resumo}

O objetivo deste trabalho foi analisar e comparar os atos incorretos dos docentes, e seus motivos, que foram percebidos pelos alunos de primeiro ano de medicina das universidades de Buenos Aires (UBA) e Complutense de Madrid (UCM), utilizando uma pesquisa com itens fechados e abertos, com o intuito de relatar o incidente e indicar o seu motivo. As categorias do incidente mais destacadas nos dois grupos foram: maltrato verbal, falta de interesse pelo aluno e avaliação injusta. Os motivos mais indicados: arrogância e falta de compromisso. $79.1 \%$ dos alunos da UCM manifestaram que o incidente ocorreu em ensino-aprendizado; e $68.1 \%$ dos da UBA, em avaliação. É interessante observar que em as duas universidades os estudantes perceberam o maltrato, embora não se perguntara sobre isso. Os resultados indicariam a necessidade de reforçar o compromisso dos docentes quanto ao respeito, a responsabilidade e a justiça.

Palavras chave: relação docente-aluno, educação médica, principalismo. 


\title{
Actions of professors considered as incorrect by medicine students:
} comparative analysis between two universities

\begin{abstract}
The purpose was to analyze and compare which incorrect actions by the professors and their motives were perceived by the students of the first year in medical school at the Buenos Aires University (UBA) and the Complutense University of Madrid (UCM). A survey with open-ended and closedended items was used in order to relate the incident and indicate its motive. The categories of the most mentioned incident in both groups were: verbal abuse, lack of interest in the student and unfair assessment. The most indicated motives: arrogance and lack of commitment. $79.1 \%$ of the UCM students stated that the incident occurred during teaching-learning; and $68.1 \%$ of the UBA students, during assessment. It is interesting to note that in both universities the students perceived abuse, in spite of not being asked in this regard. The results indicate the need to reinforce the commitment of the professors with regard to respect, responsibility and fairness.
\end{abstract}

Key words: professor-student relationship, medical education, principalism. 


\section{Introducción}

La relación docente-alumno en educación médica podrían ser la base, entre otros aspectos, de los logros del aprendizaje y de los vínculos que entable el alumno y futuro profesional con el paciente (Larkin y Mello, 2010).

Los cuatro principios que postula la teoría bioética Principalismo para la relación médicopaciente, pueden ser aplicados a la relación docente-alumno en educación médica: beneficencia, no-maleficencia, respeto por la autonomía y justicia. El primer principio se fundamenta en la obligación que tiene el docente de ayudar al alumno al logro del aprendizaje. En relación con este principio se halla el de no-maleficencia: no causar daño a los alumnos ya sea por medio de actos verbales (maltrato), de engaño o del no cumplimiento de los objetivos, y de la planificación y organización de sus actividades educativas. El principio de autonomía debe otorgar al estudiante la oportunidad de participar en decisiones que conciernen a sus objetivos y proyectos respetando sus características personales, limitaciones, valores y creencias, exigiendo a su vez un estudiante competente, responsable y maduro. En cuanto al principio de justicia, el docente debe favorecer el aprendizaje de todos sin discriminación de ninguna índole y a su vez, tener criterios justos tanto en aprendizaje como en evaluación (Beauchamp y Childress, 1994; Lifshitz, 2000).

En la actualidad se comprobó en esta relación actos incorrectos: por parte de los alumnos, principalmente falta de honestidad (Gitanjali, 2004; Kukolja et al., 2010), y por parte de los docentes, maltrato. En este último aspecto, se realizaron trabajos en distintas facultades de Medicina del mundo, en los que se analizaron diferentes tipos de maltrato percibido por los estudiantes (verbal, académico, físico y sexual), severidad y consecuencias (Oancia et al., 2000; Rautio et al., 2005; Musselman et al., 2005; Ogden et al., 2005; Wilkinson et al.,
2006; Frank et al., 2006; Haglund et al., 2009). También se investigó sobre esta temática en facultades de América Latina (Iglesias Benavídez et al., 2005; Mariscal Palle et al., 2007; Maida et al., 2006). El maltrato podría considerarse como acto incorrecto hacia el alumno, pero asimismo los estudiantes podrían percibir como incorrectos otros hechos realizados por los educadores. La percepción de estos actos puede constituir un hecho subjetivo y cada alumno lo puede interpretar de manera diferente de acuerdo con sus características personales, valores y creencias, dependiendo también del contexto en el cual se efectúe. También la percepción de un incidente incorrecto puede diferir entre los distintos estudiantes, y entre alumnos de diferentes lugares y facultades de Medicina (Bourgeois et al., 1993; Satterwhite et al., 2005; Iglesias Benavídez et al., 2005; Maida et al., 2006).

Hemos considerado como acto incorrecto aquellas acciones realizadas por los docentes que los alumnos juzgan o perciben como malas de acuerdo con sus valores personales y/o normas que creen que se deben cumplir en educación médica (Beauchamp y Childress, 1994).

Si bien los trabajos que tratan del maltrato fueron realizados en diferentes lugares del mundo, ninguno compara estos aspectos en dos facultades tradicionales de dos países de habla hispana, de reconocido prestigio académico y de similar curriculum, pero diferente cantidad de alumnos, historia, desarrollo, contexto cultural y estrategias de enseñanza-aprendizaje y de evaluación. Por ello, el primer objetivo de este trabajo fue analizar y comparar qué actos incorrectos realizados por los docentes percibieron los alumnos de primer año de las facultades de Medicina de las universidades de Buenos Aires (UBA), Argentina y Complutense de Madrid (UCM), España, teniendo en cuenta la instancia en que se produjeron. Un segundo objetivo fue analizar y comparar los diferentes motivos de dichos actos que fueron percibidos por los alumnos de ambas universidades. 


\section{Material y método}

Esta investigación se basa en un diseño seccional explicativo de comparación de grupos (alumnos de primer año de la carrera de Medicina de la UBA y la UCM), con el objeto de comparar similitudes y discrepancias respecto de la percepción de los alumnos de actos incorrectos realizados por los docentes, teniendo en cuenta las diferencias de ambas instituciones en cuanto al número de alumnos y estrategias de enseñanza-aprendizaje y evaluación, principalmente (Sierra Bravo, 1998).

Una encuesta anónima y voluntaria fue administrada a los alumnos de primer año de tres comisiones de la UBA y dos comisiones de la UCM. Se utilizó un muestreo de grupo seleccionando los mismos en forma aleatoria, entre las distintas comisiones de estudiantes de primer año y de los diferentes turnos (mañana, tarde y noche para la UBA y mañana y tarde para la UCM, de acuerdo con el horario de las cursadas) (Pagano y Gauvreau, 2001). La encuesta fue administrada en el inicio de una clase, utilizando cinco minutos para una breve explicación y diez minutos para completar los distintos ítems.

Dicha encuesta presenta una introducción (objetivos e indicaciones), tres ítems cerrados: género y edad del alumno, e instancia en que se produjo: proceso de enseñanza-aprendizaje (clases teóricas, trabajos prácticos, laboratorios, seminarios, etc.) o de evaluación (orales, escritas), y dos ítems abiertos: narrar un acto incorrecto describiendo sus circunstancias y señalar los motivos que creen que lo produjeron. Se utilizó terminología general y no referida a ningún acto incorrecto específico. No se dio ninguna definición ni caracterización, teniendo como propósito que el alumno tuviese libertad para señalar cualquier tipo de acto que haya percibido como incorrecto (Satterwhite et al., 1998). Tanto la investigación como la encuesta fueron aprobadas por el Comité de Ética de un hospital asociado a la Facultad de Medicina de la UBA.
Las cuatro primeras autoras analizaron los ítems abiertos buscando acciones y motivos semejantes, para construir categorías que facilitaran la clasificación. Luego, se analizó el contenido para clasificar el acto y las razones del mismo. Se tuvo una discrepancia del 5\%, llegando luego de un segundo análisis en conjunto a un acuerdo total. No hubo validación formal de la encuesta misma, pero los datos fueron analizados con base en la consistencia interna de los mismos (Silver y Glicken, 1990). Las categorías del incidente incorrecto fueron:

- Maltrato verbal: humillación, denigración y subestimación.

- Desinterés por el alumno: 1legadas tarde o ausentismo, retirarse del salón ante la no respuesta de los alumnos, fumar en el aula, no responder inquietudes, hablar por celular, no mirar al alumno en un examen oral.

- Evaluación injusta: falta de criterio, dando importancia a contenidos específicos o que no corresponden al programa o preguntas imprecisas o confusas.

- Discriminación o ridiculización: por género, edad, vestimenta y burla ante respuestas incorrectas de los alumnos.

- Comentarios o humor sexual: chistes, palabras con doble sentido, señalar partes determinadas del cuerpo.

- Falta de habilidades pedagógicas: falta de planificación y organización de la clase, nula organización y lectura de los Power Point, falta de criterio para selección de contenidos, no exponer los conocimientos mínimos, cometer errores a propósito.

- Maltrato fisico: golpear, pegar, arrojar algún elemento.

En cuanto a los motivos, se determinaron las siguientes categorías que se clasificaron en inherentes al docente, a la relación docente-alumno, al alumno, a la institución y no justificables. 
Los motivos inherentes al docente se caracterizaron como:

- Soberbia: altanería, altivez, arrogancia, autoritarismo.

- Falta de preparación pedagógica: no saber planificar y organizar una clase o prueba evaluativa, incompetencia, falta de criterio al evaluar, métodos inadecuados de enseñanza.

- Falta de interés por el alumno: pereza del docente, falta de ganas de dar clases, aburrido de la docencia, falta de compromiso como docente, falta de atención, no escuchar al alumno.

- Falta de respeto por el alumno: mala educación, falta de valores.

- Sobre exigencia al alumno: buscar la excelencia, máximo rendimiento, formar una élite, no aprobar a nadie, normas rígidas.

- Excentricidad: llamar la atención, exceso de confianza, no tener vergüenza.

- Problemas personales: familiares, laborales, económicos.

- Cansancio: estrés, mucha actividad, pocas horas de sueño.
Para el análisis de los datos se construyó una base donde se volcó la información de los ítems. Se efectuó el análisis de los mismos estableciéndose relaciones entre las respuestas de ambos grupos para las categorías de actos y motivos, comprobándose si existía relación significativa utilizando el test no paramétrico $\mathrm{X}^{2}(\mathrm{P}<0,05)$.

\section{Resultados}

Los alumnos que completaron la encuesta fueron 217 de la UBA y 161 de la UCM. De los primeros, el $68.7 \%$ de los alumnos fueron mujeres y tuvieron un promedio de edad de $21.44(\mathrm{DE}=3.46)$, mientras que en el segundo grupo el género femenino representó el $73.9 \%$, y un promedio de edad de 19.39 $(\mathrm{DE}=1.57)$.

Al comparar las respuestas de los alumnos de la UBA con los de la UCM observamos que estos últimos señalaron mayor porcentaje de acciones incorrectas en el proceso de enseñanza-aprendizaje (79.1\%), mientras que los estudiantes de la UBA los indicaron en el proceso de evaluación (68.1\%) (véase gráfica 1) $(\mathrm{p}<0.05)$.

\section{Gráfica 1. Comparación entre los alumnos de UBA y de la UCM según instancia del incidente}

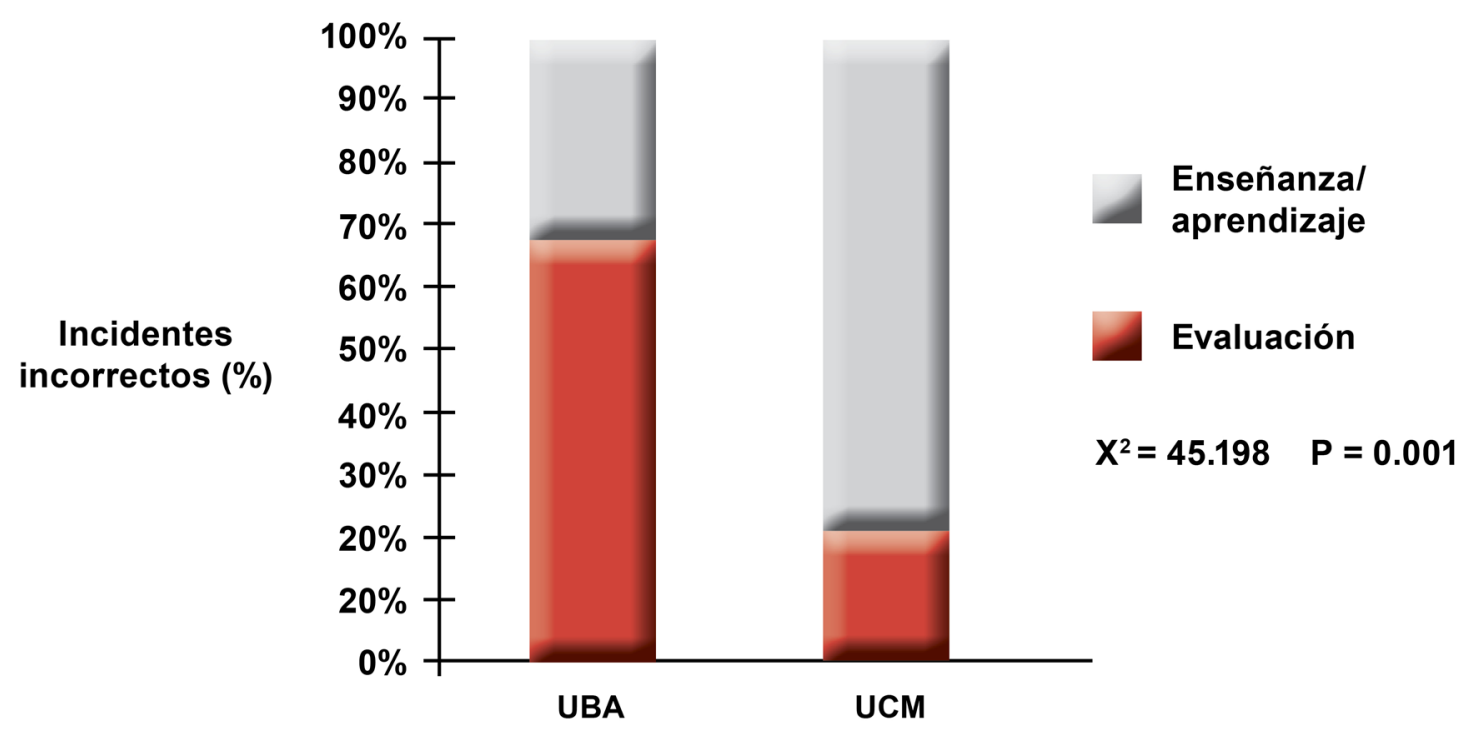


Al analizar las categorías de los actos incorrectos se encontró relación significativa entre las respuestas de los alumnos de ambas universidades. El porcentaje de actos referidos a "maltrato verbal" señalado por los estudiantes de la UBA fue mayor (43.4\%), comparado con el de la UCM $(32.2 \%)$ (véase gráfica 2$)$.

Una alumna se refirió al "maltrato verbal" en los siguientes términos:

El ayudante actuó de manera inapropiada, tratándome de ignorante y que no sabía cómo había pasado primer grado, también me dijo que no servía como futura médica y mucho menos como anatomista, entre otras cosas.

La categoría "desinterés por el alumno" fue señalada en igual porcentaje que "maltrato verbal" (32.2\%) por los estudiantes de esta última facultad, mientras que la segunda categoría más señalada por los estudiantes de la UBA fue "evaluación injusta" (20.5\%) (véase gráfica 2).

El "desinterés por el alumno" fue percibido por un alumno al manifestar:

Un profesor llegó tarde a clase. No se le escuchaba bien con el micrófono, así que no sabíamos lo que estaba diciendo. El preguntó: “¿Hay alguien en clase?" Nosotros no sabíamos qué había dicho, así que no contestamos. Lo preguntó dos veces y decidió abandonar la clase y esa parte de la teoría no volvió a ser dada.

En cuanto a la "evaluación injusta" fue expresada de la siguiente manera:

En el práctico del parcial me tomaron los preparados en frascos. Contesté bien todos los números

\section{Gráfica 2. Comparación entre los alumnos de UBA y de la UCM según categoría del incidente}

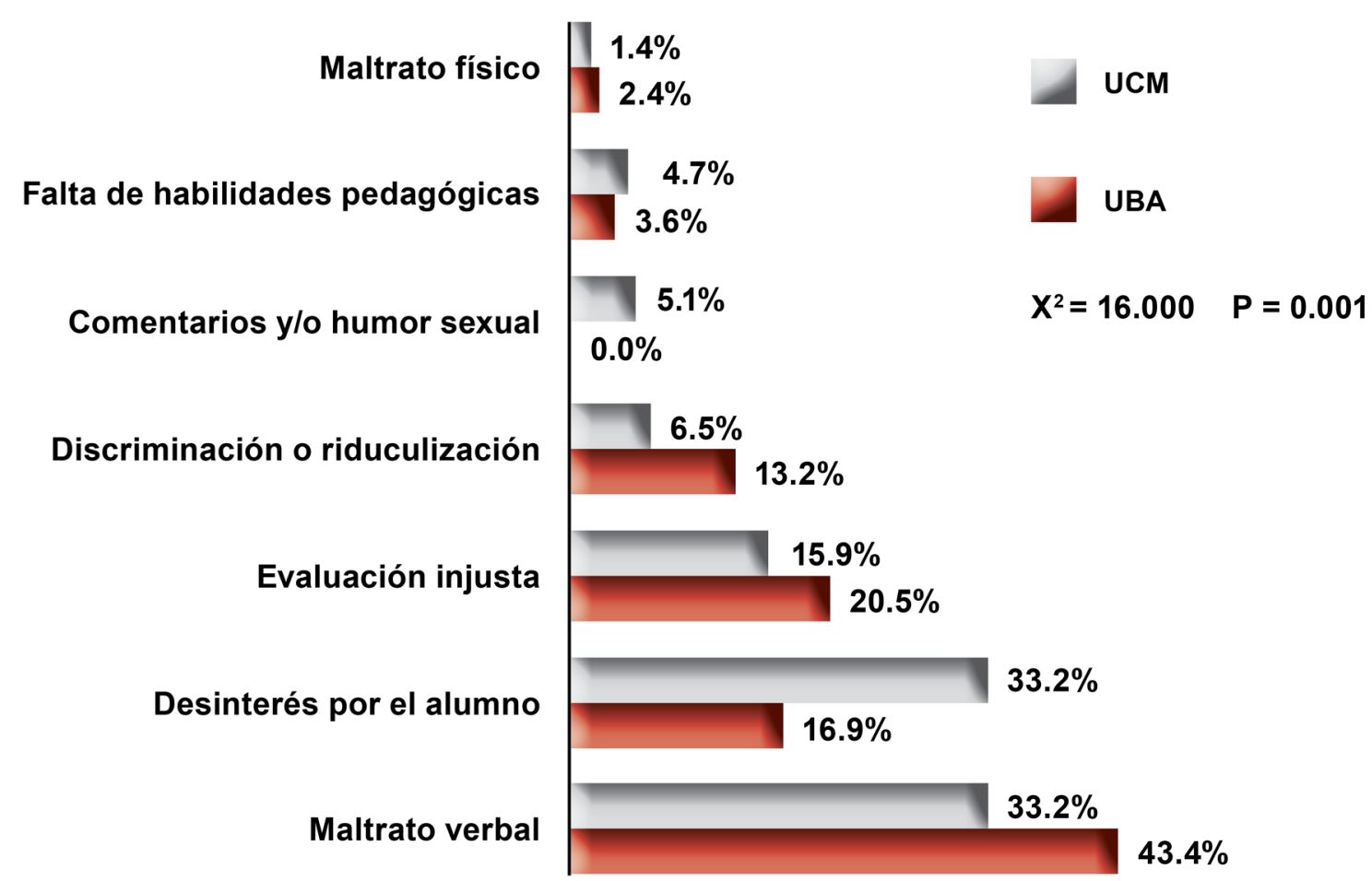


que me preguntaba. Cuando llegué a las radiografías me dijo que me ponía un 3 por no haber hablado mucho. Le dije que no me había equivocado y lo aceptó. Pero contestó que el parcial hubiese sido mejor si yo hubiera hablado sola, sin que me pregunte.

La cuarta categoría manifestada fue la "discriminación o ridiculización", siendo los alumnos de la UBA los que lo señalan en mayor medida (13.2\%) con respecto a los de la UCM (6.5\%) (véase gráfica 2). En referencia a la "discriminación" por género un alumno expresó:

En los exámenes parciales, varios ayudantes tienen como criterio el aspecto físico y la buena presencia de las alumnas evaluadas, sepan o no los contenidos teóricos de la materia. Motivo por el que corren en desventaja los alumnos masculinos o poco atractivos.

Sólo los alumnos de la UCM señalaron algunos actos referidos a "comentarios o humor sexual" (5.1\%). La categoría "falta de habilidades pedagógicas" tuvo menor porcentaje para los alumnos de ambas instituciones (véase gráfica 2). Un alumno expresó:

El acto apunta a una cierta regularidad con la que algunos docentes parecieran no sentir la vocación que, se supone, los llevó a elegir ocupar ese puesto en la estructura educativa. Pretendería una actitud más propicia para poder lograr el objetivo, que sería orientar al alumno de la mejor forma posible en su camino educativo.

El "maltrato físico" fue percibido por muy pocos en ambos grupos (gráfica 2). Ejemplo de ello, es lo que manifestó un alumno:

En el parcial entregué la hoja en la que había que especificar los órganos diagnosticados. La hoja estaba escrita en lápiz, motivo que provocó un desagrado en el ayudante. La reacción fue tirarme de mala gana la hoja y una lapicera.

Cuando se consultó a los alumnos sobre cuáles eran los motivos que creían que habían provocado el acto, los alumnos de la UCM señalaron mayor número de razones comparados con los alumnos de la UBA. En ambos grupos la mayoría indicó razones referidas al "propio docente" y en menor medida, a la "relación docente alumno" (malentendido, fallas de comunicación, poco contacto), al "estudiante" (apatía, miedo al profesor, falta de estudio) y a la "institución" (desorganización académica, problemas técnicos, errores administrativos), con porcentajes similares en ambos grupos. Esta relación no resultó significativa (gráfica 3).

Al comparar específicamente los motivos propios del docente, la relación resultó significativa. La razón más señalada por ambos grupos fue la "soberbia". Se diferenciaron en "falta de compromiso" y "sobre exigencia al alumno". Los estudiantes de la UBA señalaron en una cuarta parte que los motivos se debieron a la "falta de preparación pedagógica" del docente (véase gráfica 4).

En síntesis, para los alumnos de la UCM los actos incorrectos ocurrieron en la instancia de enseñanza-aprendizaje, mientras que los estudiantes de la UBA indicaron que ocurrió en el proceso de evaluación, siendo el "maltrato verbal" y el "desinterés por el alumno" las categorías más señaladas por los alumnos de ambas facultades. Categorías que, según los estudiantes, fueron motivadas por la "soberbia" del docente.

\section{Discusión}

La relación docente-alumno en educación médica es fundamental para el vínculo que entablará el estudiante y profesional con el paciente. El comportamiento del docente es un modelo para la 


\section{Gráfica 3. Comparación entre los alumnos de UBA y de la UCM} según motivos del incidente

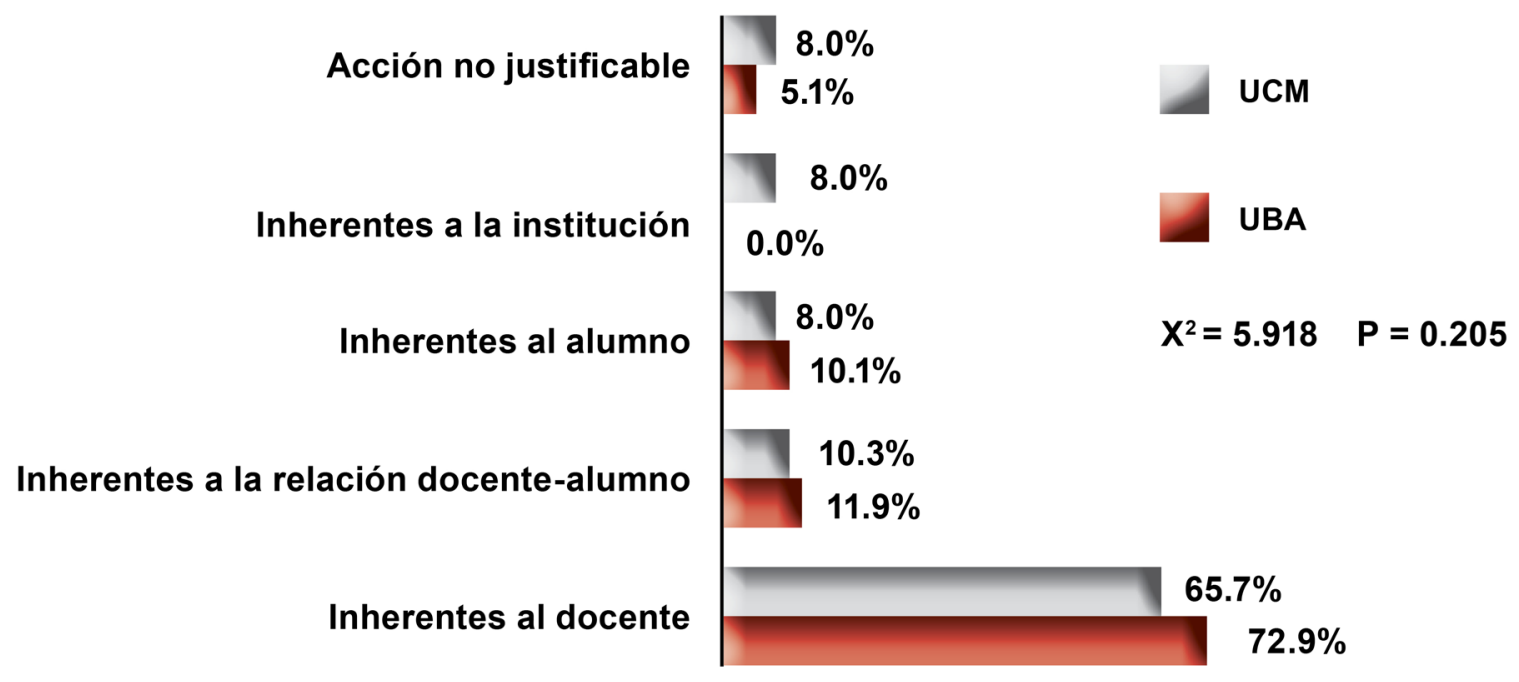

13 alumnos de la UCM y 14 de UBA no contestaron o no saben el motivo

Gráfica 4. Comparación entre los alumnos de UBA y de la UCM según motivos del incidente inherentes al docente

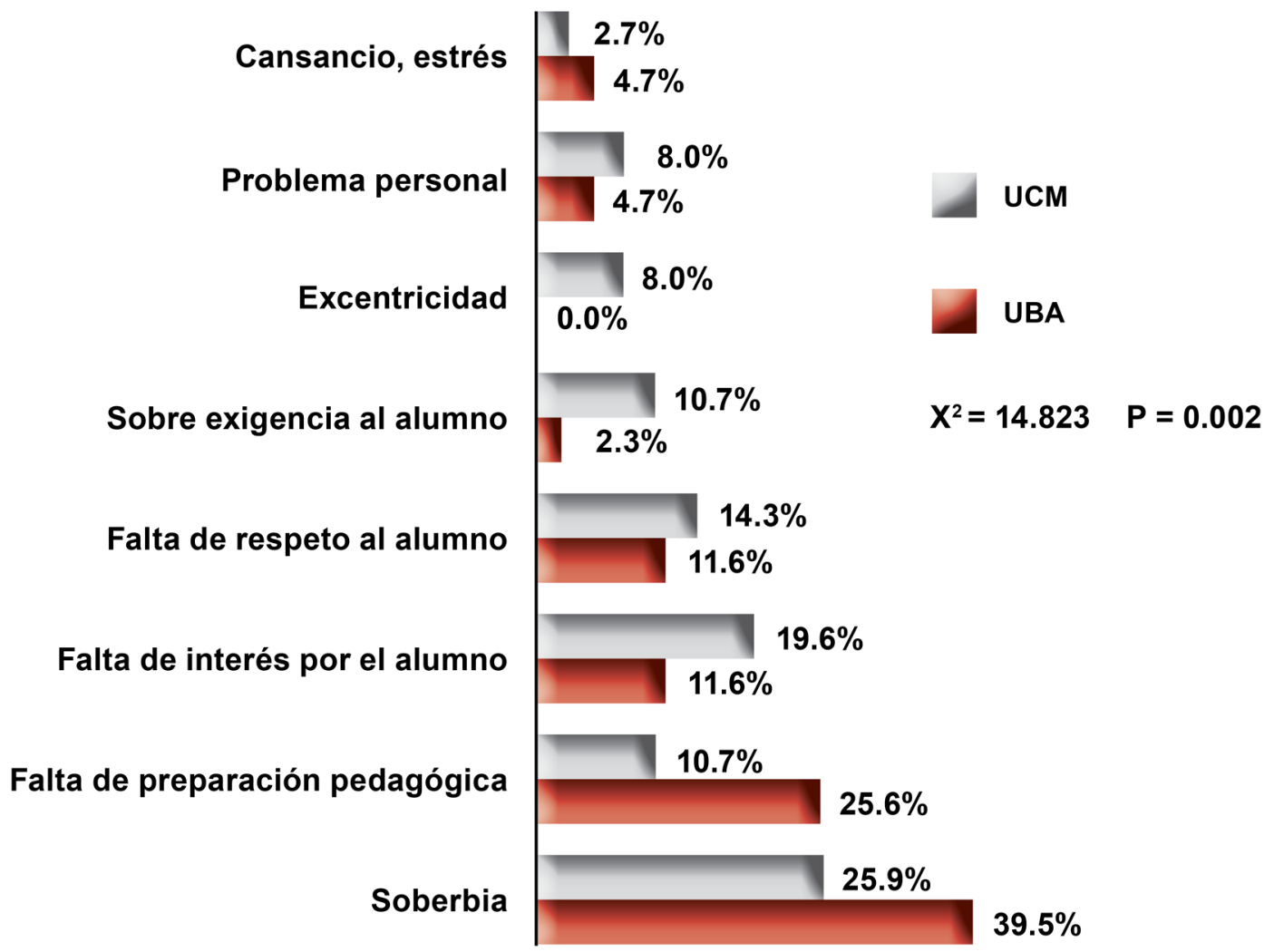


formación del alumno basada principalmente en los principios bioéticos (Beauchamp y Childress, 1994; Lifshitz, 2000; Larkin y Mello, 2010).

Las diferencias entre los dos grupos en cuanto a la instancia en que se produjo el acto (enseñanzaaprendizaje o evaluación) quizás pueda deberse a las diferentes metodologías utilizadas, como también a las distintas características y exigencia del curriculum de la carrera. En la UBA hay predominio de pruebas orales finales y parciales en las materias de primer año, donde la relación es cara a cara. Muchas veces son los auxiliares docentes los encargados de estas pruebas; éstos, por lo general, no cuentan con los recursos pedagógicos necesarios. Pueden colaborar también, dado el gran número de estudiantes en estas evaluaciones, otros profesores que no tuvieron previo contacto con los alumnos. En cambio, en la UCM las pruebas son escritas y de selección múltiple, motivando menos conflictos personales. También al haber menor cantidad de alumnos en esta última institución, el contacto con el docente podría ser más cercano y propiciar el desarrollo de estos actos en las actividades de enseñanza-aprendizaje.

Algunos de los artículos señalados incluyen definiciones o caracterizaciones de la terminología dada a los estudiantes (Ogden et al., 2005; Satterwhite et al., 1998), mientras que en otros, los alumnos hacen sus propias interpretaciones de la terminología empleada (Frank et al., 2006; Lubitz y Nguyen, 1996). Como ya hemos establecido en nuestro estudio, se les solicitó a los estudiantes una cuestión general y no se les dio ninguna definición. Estos resultados obtenidos no se pueden comparar con ninguno de los trabajos realizados, dado que los mismos a nivel mundial se refieren al "maltrato" específicamente, mientras que el presente hace referencia a un concepto más amplio y general. A pesar de ello, el "maltrato" fue la categoría más señalada, en porcentaje inferior al indicado en estos trabajos (Rautio et al., 2005;
Musselman et al., 2005; Wilkinson et al., 2006; Haglund et al., 2009; Iglesias Benavídez et al., 2005; Maida et al., 2006; Al-Hussain et al., 2008; Rancich et al., 2008; Martinez y Lo, 2006). El "maltrato verbal" fue el más común en ambos grupos, al igual que en otros artículos (Oancia et al., 2000; Musselman et al., 2005; Ogden et al., 2005). Estos actos docentes de maltrato percibidos por los estudiantes estarían infringiendo el principio de no-maleficencia por las consecuencias de distinta índole que podrían ocasionar a los estudiantes: ansiedad, estrés, bajo rendimiento, abandono de estudios, reprobación de materias, dificultades en las relaciones, entre otras (Beauchamp y Childress, 1994; Lifshitz, 2000).

La segunda categoría manifestada fue la "evaluación injusta". Ésta puede generar de por sí un conflicto. Para el docente implica determinar criterios objetivos y justos de evaluación, acordes con fines y contenidos de la materia (aplicación del principio de justicia) (Beauchamp y Childress, 1994; Lifshitz, 2000). Para el estudiante podrían ser situaciones de estrés y ansiedad, que algunas veces ponen en juego una materia o un año de la carrera.

Las conductas de "desinterés por el alumno" podrían estar relacionadas con la falta de un modelo a seguir para la práctica de la docencia, y con la circunstancia de que muchos docentes trabajan ad honorem en la UBA, lo que podría reflejarse como un menor compromiso de responsabilidad con la facultad y los alumnos, no cumpliendo así con el principio de beneficencia (Beauchamp y Childress, 1994; Lifshitz, 2000).

Respecto de los actos relacionados con "discriminación", la mayoría correspondió a género, y referidos por varones y mujeres, a diferencia de otros trabajos donde la mujer señaló más "maltrato" (Oancia et al., 2000; Rautio et al., 2005; Iglesias Benavídez et al., 2005; Dyrbye et al., 2005), excepto en una publicación (Al-Hussain et al., 2008). En este aspecto, también las conductas percibidas por 
los alumnos estarían en relación con el principio de justicia, dado que el docente debe respetar las individualidades y características personales del alumno, no haciendo ningún tipo de diferenciación en el trato ((Beauchamp y Childress, 1994; Lifshitz, 2000).

Sólo los alumnos de la UCM señalaron en muy bajo porcentaje "comentarios o humor sexual". Estos resultados pueden deberse a que estos hechos son de índole privada y pueden significar vergüenza o denigración.

En ninguno de los trabajos que analizan el "maltrato" de los docentes, solicitan a los alumnos que señalen cuáles consideran que pueden ser los motivos que llevaron al docente a realizar esta acción.

Si bien los alumnos de la UCM indicaron más cantidad de motivos, la mayoría en ambos grupos fueron referidos al "propio docente" y en menor medida a la "relación docente-alumno", al "propio alumno" o a "problemas institucionales", siendo estas últimas categorías más manifestadas por los alumnos de la UCM. Esto se puede deber a la distinta estructura administrativa y edilicia de la facultad y a la diferencia en el número de alumnos. Además, es poco probable que el alumno se culpe a sí mismo, porque narra un hecho en el que se sintió afectado.

En ambos grupos, la "soberbia" fue la razón más señalada, con mucho mayor predominio en la UBA, mientras que los alumnos de la UCM hacen hincapié en la "falta de compromiso con el estudiante". La "soberbia" puede deberse a que el docente está en una situación de mayor jerarquía, tradicional en Medicina y puede manifestarla a través del "maltrato" (Oancia et al., 2000; Musselman et al., 2005; Ogden et al., 2005). Esta actitud del docente demuestra una actitud paternalista autoritaria, tradicional en educación médica, contraria a la autonomía del alumno, a pesar de que en la actualidad las tendencias modernas centran el proceso educativo en el estudiante (Beauchamp y Childress, 1994; Lifshitz, 2000).

Indudablemente, estos conflictos deben haber traído consecuencias a los alumnos (angustia, depresión, dudas en su vocación, mal rendimiento, reprobación de materias, dudas en cuanto a seguir la carrera, etc.), dependiendo de las características del sujeto. Estos aspectos no fueron indagados y constituyen limitaciones del trabajo, dado que en otros artículos se tuvieron en cuenta (Oancia et al., 2000; Frank et al., 2006; Satterwhite et al., 1998) y deberán formar parte de futuras investigaciones.

Este estudio investigó las percepciones de los alumnos sobre los actos incorrectos, constituyendo las mismas interpretaciones de hechos subjetivos, por ende, no se usó ningún criterio objetivo para validar estos episodios (Silver y Glicken, 1990; Wolf et al., 1991).

Si bien los datos obtenidos sugieren la presencia de algunos hallazgos interesantes en cuanto a las diferencias entre los alumnos de las dos universidades, debemos ser cautos, ya que pertenecen sólo a estudiantes del primer año. Además, la narración de un acto pasado puede llevar al estudiante a una sub o sobrevaloración del hecho, reflejando actitudes, valores y criterios de jerarquización, perdiendo objetividad, como es señalado en otros trabajos (Oancia et al., 2000; Frank et al., 2006; Satterwhite et al., 1998).

\section{Conclusiones}

En conclusión, los alumnos de ambas universidades percibieron tanto actos referidos a compromisos que afectan a toda relación humana (respeto, interés y justicia), como también compromisos referidos a los procesos de enseñanza aprendizaje y de evaluación en educación médica (evaluación injusta, falta de habilidades pedagógicas). Los motivos señalados por los estudiantes de ambos grupos fueron en su mayoría referidos a las características personales del educador. 
Por ello, al ser esta relación docente-alumno fundamental para el desarrollo del profesional, debería estar basada en los principios de beneficencia, no-maleficencia, respeto por la autonomía y justicia, constituyendo un modelo para la formación.

\section{Referencias}

Al-Hussain, S., M. Al-Haidari, N. Kouri et al. (2008), "Prevalence of mistreatment and justice of grading system in five health related faculties in Jordan University of Science and Technology", en Medical Teacher, núm. 30, pp. 82-86.

Beauchamp, T., J. Childress (1994), Principles of biomedical ethics, Nueva York, Oxford University Press.

Bourgeois J., J. Kay, J. Rudisill, D. Bienenfeld et al. (1993), "Medical student abuse: perceptions and experience", en Medical Education, núm. 27, pp. 363-370.

Dyrbye, L., M. Thomas, T. Shanafelt (2005), "Medical student sistress causes, consequences, and proposed solutions", en Mayo Clinic Proccedings, núm. 80, pp. 1613-1622.

Frank, E., J. Carrera, T. Stratton et al. (2006), "Experiences of belittlement and harassment and their correlates among medical students in the United States: longitudinal survey", en British Medical Journal, núm. 333, pp. 682, http://dx.doi. org/10.1136/bmj.38924.722037.7C [consulta: marzo 2009].

Gitanjali, B. (2004), "Academic dishonesty in Indian medical colleges", en Journal of Postgraduate Medicine, núm. 50, pp. 281-284.

Haglund, M., M. Aan Het Rot, N. Cooper et al. (2009), "Resilience in the third year of medical school: a prospective study of the associations between stressful events occurring during clinical rotations and student well-being", en Academic Medicine, núm.
84, pp. 258-268.

Iglesias Benavides, J., D. Saldívar Rodríguez, V. Bermúdez Barba et al. (2005), "Maltrato del estudiante de medicina. Percepción de 404 alumnos de cuarto, quinto y sexto años de la carrera", en Medicina Universitaria, núm. 7, pp. 191-202.

Kukolja Taradi, S., M. Taradi, T. Knezevic et al. (2010), "Students come to medical schools prepared to cheat: a multi-campus investigation", en Journal of Medical Ethics, núm. 36, pp. 666-670.

Larkin, G. y M. Mello (2010), "Commentary: doctors without boundaries: the ethics of teacher-student relationships in academic medicine", en Academic Medicine, núm. 85, pp. 752-755.

Lifshitz, A. (2000), "Ética en la docencia médica", en Gaceta Médica de México, núm. 136, pp. 398-404.

Lubitz, R. y D. Nguyen (1996), "Medical student abuse during third-year clerkships", en Journal of American Medical Association, núm. 275, pp. 414-416.

Maida, S., M. Herskovic, S. Pereira et al. (2006), "Percepción de conductas abusivas en estudiantes de Medicina”, en Revista Médica de Chile, núm. 134, pp. 1516-1523.

Mariscal Palle, E., O. Navia Molina, J. Paniagua et al. (2007), "Maltrato y/o violencia: fenómeno de estudio en centros de enseñanza asistenciales: Internado Rotatorio de Medicina gestión 20052006", en Cuadernos del Hospital de Clínicas, núm. 52, pp. 46-54.

Martinez, W. y B. Lo (2006), "Medical students' 
experiences with medical errors: an analysis of medical student essays", en Medical Education, núm. 42, pp. 733-741.

Musselman, L., H. Macrae, R. Reznick et al. (2005), "'You learn better under the gun': intimidation and harassment in surgical education", en Medical Education, núm. 39, pp. 926-934.

Oancia, T., C. Bohm, T. Carry et al. (2000), "The influence of gender and specialty on reporting of abusive and discriminatory behaviour by medical students, residents and physician teachers", en Medical Education, núm. 34, pp. 250-256.

Ogden, P., E. Wu, M. Elnicki et al. (2005), "Do attending physicians, nurses, residents, and medical students agree on what constitutes medical student abuse?, en Academic Medicine, núm. 80, pp. S580-S583.

Pagano, M. y K. Gauvreau (2001), Fundamentos de Bioestadística, México, Thomson Editores, pp. 517.

Rancich, A., M. Donato, R. Gelpi (2008), "Educación médica: actos en la relación docente-alumno", en Revista Argentina de Educación Médica, núm. 2, pp. 4-7. Rautio, A., V. Sunnari, M. Nuutinen et al. (2005),
"Mistreatment of university students most common during medical studies", en BMC Medical Education, núm. 5, 36, http://www.biomedcentral.com/content/ pdf/1472-6920-5-36.pdf [consulta: junio 2009].

Satterwhite, W., R. Satterwhite, C. Enarson (1998), "Medical students perceptions of unethical conduct at one medical school", en Academic Medicine, núm. 73, pp. 529-531.

Sierra Bravo, R. (1998), Técnicas de investigación social. Teoría y ejercicios, Madrid, Editorial Paraninfo.

Silver, H K. y A. Glicken (1990) "Medical student abuse: incidence, severtity and significance", en Journal of American Medical Association, núm. 263, pp. 527-532.

Wilkinson, T., D. Gill, J. Fitzjohn et al. (2006), "The impact on students of adverse experiences during medical school", en Medical Teacher, núm. 8, pp. 129-135.

Wolf, T., H. Randall, K. Von Almen et al. (1991), "Perceived mistreatment and attitude change by graduating medical students: a retrospective study", en Medical Education, núm. 25, pp. 182-190.

\section{Cómo citar este artículo:}

Rancich, Ana-María, Luciana-Yesica Niz, María-Paula Caprara et al. (2013), "Actuaciones docentes consideradas como incorrectas por los alumnos de Medicina: análisis comparativo entre dos universidades", en Revista Iberoamericana de Educación Superior (RIES), México, UNAM-IISUE/Universia, vol. IV, núm. 9, pp. 95-107, http://ries. universia.net/index.php/ries/article/view/209/html_42 [consulta: fecha de última consulta]. 